\title{
Simulation on The Mathematical Model for the Control Of Hepatitis B Virus-Hepatitis D Virus (HBV-HDV) Co-infection Transmission Dynamics in a Given Population
}

\author{
Remigius Okeke Aja ${ }^{1 *}$, Titus Ifeanyi Chinebu ${ }^{1}$ and Godwin Christopher E. Mbah ${ }^{2}$ \\ ${ }^{1}$ Department of Mathematics, Michael Okpara University of Agriculture, Umudike, Nigeria \\ ${ }^{2}$ Department of Pure and Applied Sciences, Federal College of Dental Technology and Therapy, Enugu, Nigeria \\ ${ }^{3}$ Department of Mathematics, University of Nigeria, Nsukka, Nigeria \\ * Corresponding author
}

\author{
Article Info \\ Keywords: Co-infection, Controls, Hep- \\ atitis B virus, Hepatitis D virus, Matlab, \\ Simulation. \\ 2010 AMS: 92-10, 92B05, 92C60, \\ 92D30, 93-10 \\ Received: 2 June 2021 \\ Accepted: 31 August 2021 \\ Available online: 2 September 2021
}

\begin{abstract}
This paper investigates the impact of the various parameters of the mathematical model for Hepatitis B virus-Hepatitis D virus (HBV-HDV) co-infection with controls (awareness, vaccine and therapy). It establishes that the model is biologically meaningful and epidemiologically well posed. Furthermore, simulations are carried out on the equations of the model using MATLAB and the results indicate that; when $c_{1}$ (awareness) increase from 0.08 to 0.70 , then the number of exposed HB individuals in the population will also increase. Conversely, we notice a drastic decrease in the number of exposed HBD individuals in the population when $c_{1}$ (awareness) increase from 0.08 to 0.70 . Again, we observe a decrease in the number of exposed treated individuals in the population when $c$ (therapy) increase from 0.08 to 0.50 . Similarly, we notice an increase in the number of recovered HBD individuals in the population upon the increase of $c$ (therapy) from 0.08 to 0.50 . We therefore conclude that awareness, vaccine and therapy are good measure which can be used to effectively control HBV-HDV co-infection in a population. However, awareness and vaccine are better control strategies than therapy. Hence, these simulation results provide the best framework for the control of the disease; Hepatitis B virus-Hepatitis D virus (HBV-HDV) co-infection in a population.
\end{abstract}

\section{Introduction}

Hepatitis B virus (HBV) and Hepatitis D virus (HDV) infections are major health problem. HBV virus is a DNA virus from hepadnaviridae family. Infected person or asymptomatic carriers with viral HBV are only reservoir of infection [1]. Researches show us that world prevalence of Hepatitis B surface antigen ( $\mathrm{HBsAg}$ ) carriers is from $0.1-20 \%$ with high percentage in tropical countries [1]. The risk of transmission of $\mathrm{HBV}$ via blood and blood product is much higher than Hepatitis $\mathrm{C}(\mathrm{HCV})$ and $\mathrm{HIV}$. HBV may induce chronic hepatitis that could progress to cirrhosis and hepatocellurar carcinoma [2].

Hepatitis D virus (HDV) sometimes called Hepatitis Delta Virus, was detected by Rizzetto among patients with a severe form of Hepatitis B virus (HBV) infection in the year of 1977 [3]. Chronic Hepatitis D still remains a major cause of liver transplantation and death [4]. Cirrhosis the final stage of chronic Hepatitis, has been considered to be irreversible [5]. HDV induces a broad range of clinical manifestations in humans, ranging from asymptomatic cases to patients with fulminant hepatitis and hepatocellular carcinoma ( [6], [7]). HDV or Delta virus is an incomplete defective RNA virus requiring concomitant presence of HBV for its survival and replication ( [8]; [9]; [10]; [11]). Thus, HDV can replicate only in people who are also infected with HBV.

The epidemiology of HDV infection is similar to HBV with some exception ( [10]; [12]). It is estimated that approximately 5\% of the HBV carrier are co-infected with HDV infection worldwide [6]. However, the prevalence of HDV in HBV carriers varies around the world [13].

Email addresses and ORCID numbers: ajah.remigius@mouau.edu.ng, https://orcid.org/0000-0002-1697-127x (R. 0. Aja), titusifeanyi5432@gmail.com, https://orcid.org/0000-0002-2143-7733 (T. I. Chinebu),godwin.mbah@unn.edu.ng, https://orcid.org/00000002-5395-8680, https://orcid.org/0000-0002-9208-5421 (G. C. E. Mbah) 
As mentioned above, HDV is a defective virus since it cannot produce infectious virions without the help of a co-infecting helper virus. The helper virus is Hepatitis B that supplies the HBsAg surface protein. In budding out of the cell, HDV acquires a membrane containing HBsAg. It uses HBsAg as its envelope protein; thus, HBV co-infection is necessary for the packaging and release of HDV virions from infected hepatocytes. The smallest HBsAg is even sufficient to package the HDV genome.

Due to the complexity of the health problems caused by HBV infection and HDV infection as mentioned above, it becomes imperative to study the co-infection of the two deadly diseases. Thus, [14] developed a mathematical model for Hepatitis B virus - Hepatitis D virus (HBV-HDV) co-infection with controls. They obtained the basic reproduction number $R_{0}$ for the model and consequently carried out sensitivity analysis on the model using MATHCAD. [15], investigated stability analysis of Hepatitis B virus-Hepatitis D virus co-infection using Jacobian method. Valid results were obtained by evaluating the Jacobian matrix formed numerically.

This paper investigates the impact of the various parameters of the mathematical model developed by [14]. It establishes that the model is biologically meaningful and epidemiologically well posed. Furthermore, simulations are carried out on the equations of the model using MATLAB and the results are vividly discussed. From the results, awareness and vaccine are better control strategies than therapy. Thus, the results of the simulation provide the best framework for the control of HBV-HDV co-infection in a population.

\section{Model formulation}

Section 2 and its subheadings are taken from references [14] and [15]

\subsection{Assumptions of the Model}

The model is based on the following assumptions:

1. The individuals that make up the population are grouped into different compartments or groups according to their epidemiological state.

2. The population size in a compartment varies with respect to time.

3. The population mixes homogeneously. That is, all susceptible individuals are equally likely to be infected by infectious individuals if they come in contact with one another.

4. The infection does not confer immunity to the recovered individuals and so they can go back to the susceptible class at any given time.

5. The individuals in each compartment have equal natural death rate given as $\mu$.

6. The gain in the infectious class is at a rate proportional to the number of infectious and susceptible individuals. That is, $\beta S I$, where $\beta>0$ is a contact parameter (effective contact rate). The susceptible are lost at the same rate.

7. The rate of removal of infectious to the recovered or removed class is proportional to the number of infectious individuals.

\subsection{Model Variables}

The following variables are used in this study, thus:

S: The number of susceptible individuals

$\mathbf{E}_{\mathbf{H} B}$ : The number of individuals who are exposed to HBV

$\mathbf{E}_{\mathbf{T} H B}$ : The number of individuals who are exposed to HBV and are being treated

$\mathbf{I}_{\mathbf{H B}}$ : The number of individuals who are infectious of $\mathrm{HBV}$

$\mathbf{I}_{\mathbf{N H B}}$ : The number of individuals who are infectious of HBV and not being treated

$\mathbf{I}_{\mathbf{T} H B}$ : The number of individuals who are infected with HBV and are being treated

$\mathbf{R}_{\mathbf{H} B}$ : The number of individuals who have been treated of HBV and have recovered

$\mathbf{E}_{\mathbf{H} B D}$ : The number of individuals who are infectious of HBV and latently infected with HDV (exposed of HDV)

$\mathbf{E}_{\mathbf{T} H B D}$ : The number of individuals who are infectious of HBV and now exposed to HDV and being treated

$\mathbf{I}_{\mathbf{H} B D}$ : The number of individuals who are infected with HBV and HDV at the same time

$\mathbf{I}_{\mathbf{N} H B D}$ : The number of individuals who are infected with HBV and HDV and not being treated of any

$\mathbf{I}_{\mathbf{T} H B D}$ : The number of individuals who are infected with HBV and HDV and are being treated of both

$\mathbf{R}_{\mathbf{H B D}}$ : The number of individuals who have recovered from HBV-HDV co-infection after they have been treated

\subsection{Parameters of the Model}

We shall also use the following parameters in this model, thus:

$\pi$ : The number of people that enter the population (the number of individuals that enter into the susceptible class)

$\beta_{1}$ : Contact rate for $I_{H B}$ with susceptible individuals $(S)$. i.e., the rate at which individuals who had contact with HBV infectious person become exposed to HBV

$\beta_{2}$ : Contact rate for $I_{H B D}$ with susceptible classes $\left(E_{H B}, I_{N H B} a n d I_{T H B}\right)$

$\tau$ : The rate at which individuals who are exposed to HBV become infectious of HBV

$\omega$ : The rate at which individuals who are exposed to HBV enter into exposed and being treated compartment or class $\left(E_{T H B}\right)$

$\rho_{2}$ : The rate at which individuals who are infectious of HBV enter into infectious and being treated HBV class $\left(I_{T H B}\right)$

$\rho_{1}$ : The rate at which individuals who are infectious of HBV enter into infectious and not being treated of $\mathrm{HBV}\left(I_{N H B}\right)$

$\lambda_{1}$ : The rate at which individuals that are infectious and being treated of HBV goes back to exposed HBV class

$\lambda_{2}$ : The rate at which individuals who are infectious and being treated of HBV recover from HBV

$\alpha$ : The rate at which individuals who are exposed and being treated of HBV recover

$\phi_{1}$ : The rate at which individuals who recovered from HBV goes back to susceptible class

$\varphi$ : The rate at which individuals that are infectious and being treated of HBV become exposed to HDV, that is $E_{H B D}$

$\psi$ : The rate at which individuals who are infectious of $\mathrm{HBV}$ and not being treated become exposed to HDV, that is $E_{H B D}$ 
$\beta_{2}\left[1-\left(c_{1}+c_{2}\right)\right]:$ The rate at which individuals who are exposed of HBV become exposed to HDV $\left(E_{H B D}\right)$

$\zeta$ : The rate at which HBV infectious individuals who are exposed to HDV enter into exposed HDV being treated class $\left(E_{T H B D}\right)$. that is the rate at which individuals who are exposed of HBV-HDV co-infection enter into exposed HBV-HDV being treated class $\left(E_{T H B D}\right)$

$\kappa$ : The rate at which HBV infectious individuals who are exposed to HDV become infectious of HBV-HDV

$\varepsilon_{1}$ : The rate at which individuals who are infectious of HBV-HDV enter into infectious HBV-HDV not being treated class $\left(I_{N H B D}\right)$

$\varepsilon_{2}$ : The rate at which individuals who are infectious of HBV-HDV enter into infectious HBV-HDV being treated class $\left(I_{T H B D}\right)$

$\eta$ : The rate at which individuals that are infectious of HBV-HDV and are being treated recover

$\in:$ The rate at which HBV infectious individuals who are exposed to HDV and then being treated become infectious of HBV-HDV

$\theta(1+c)$ : The rate at which HBV infectious individuals that are exposed to HDV and are being treated recover

$\phi_{2}$ : The rate at which individuals who recovered from HBV-HDV goes back to susceptible class again

$\mu$ : The natural mortality/death rate

$\delta$ : HBV-induced mortality/death rate for people infectious of HBV treated class

$\delta_{1}$ : HBV-induced mortality/death rate for people infectious of HBV but not being treated class

$\delta_{2}$ : HDV-induced mortality/death rate for HBV infectious individuals who are exposed and being treated of HDV

$\delta_{3}$ : HBV-HDV-induced mortality/death rate for HBV infectious individuals who are infectious of HBV-HDV and are being treated

$\delta_{4}$ : HBV-HDV-induced mortality/death rate for individuals who are infectious of HBV-HDV and are not being treated

$\theta$ : Cure rate

$c$

$\left.\begin{array}{l}c_{1} \\ c_{2}\end{array}\right\}$ : Infectivity controls; where $c_{1}$ is awareness, $c_{2}$ is vaccine and $c$ is therapy

\subsection{Model Description}

Based on the standard SEIR model, the population is partitioned into thirteen compartments or classes namely: Susceptible $(S)$, Exposed to HBV $\left(E_{H B}\right)$, Exposed to HBV and Treated $\left(E_{T H B}\right)$, Infectious of HBV $\left(I_{H B}\right)$, Infectious of HBV and Treated $\left(I_{T H B}\right)$, Infectious of HBV and not Treated $\left(I_{N H B}\right)$, Recovered of HBV $\left(R_{H B}\right)$, HBV infectious now Exposed to HDV $\left(E_{H B D}\right)$, HBV infectious now Exposed to HDV and treated $\left(E_{T H B D}\right)$, Infectious of HBV-HDV co-infection $\left(I_{H B D}\right)$, Infectious of HBV-HDV co-infection and treated $\left(I_{T H B D}\right)$, Infectious of HBV-HDV co-infection and not treated $\left(I_{N H B D}\right)$, Recovered of HBV-HDV co-infection $\left(R_{H B D}\right)$ Compartments.

\subsection{Model Equations}

$$
\begin{aligned}
& \frac{d S}{d t}=\pi+\phi_{1} R_{H B}+\phi_{2} R_{H B D}-\beta_{1} S I_{H B}-\mu S \\
& \frac{d E_{H B}}{d t}=\beta_{1} S I_{H B}-\beta_{2}\left[1-\left(c_{1}+c_{2}\right)\right] E_{H B} I_{H B D}+\lambda_{1} I_{T H B}-\omega E_{H B}-\tau E_{H B}-\mu E_{H B} \\
& \frac{d E_{T H B}}{d t}=\omega E_{H B}-\mu E_{T H B}-\alpha E_{T H B} \\
& \frac{d I_{H B}}{d t}=\tau E_{H B}-\rho_{1} I_{H B}-\rho_{2} I_{H B} \\
& \frac{d I_{N H B}}{d t}=\rho_{1} I_{H B}-\psi I_{N H B} I_{H B D}-\mu I_{N H B}-\delta_{1} I_{N H B} \\
& \frac{d I_{T H B}}{d t}=\rho_{2} I_{H B}-\varphi I_{T H B}-\lambda_{1} I_{T H B}-\lambda_{2} I_{T H B}-\mu I_{T H B}-\delta I_{T H B} \\
& \frac{d R_{H B}}{d t}=\lambda_{2} I_{T H B}+\alpha E_{T H B}-\phi_{1} R_{H B}-\mu R_{H B} \\
& \frac{d E_{H B D}}{d t}=\beta_{2} E_{H B} I_{H B D}+\psi I_{N H B} I_{H B D}+\varphi I_{T H B} E_{H B D}-\kappa E_{H B D}-\zeta E_{H B D}-\mu E_{H B D} \\
& \frac{d E_{T H B D}}{d t}=\zeta E_{H B D}-\theta(1+c) E_{T H B D}-\in E_{T H B D}-\mu E_{T H B D}-\delta_{2} E_{T H B D} \\
& \frac{d I_{H B D}}{d t}=\kappa E_{H B D}+\in E_{T H B D}-\varepsilon_{1} I_{H B D}-\varepsilon_{2} I_{H B D} \\
& \frac{d I_{N H B D}}{d t}=\varepsilon_{1} I_{H B D}-\mu I_{N H B D}-\delta_{4} I_{N H B D}
\end{aligned}
$$




$$
\begin{gathered}
\frac{d I_{T H B D}}{d t}=\varepsilon_{2} I_{H B D}-\eta I_{T H B D}-\mu I_{T H B D}-\delta_{3} I_{T H B D} \\
\frac{d R_{H B D}}{d t}=\theta(1+c) E_{T H B D}+\eta I_{T H B D}-\mu R_{H B D}-\phi_{2} R_{H B D} \\
N=S+E_{H B}+E_{T H B}+I_{N H B}+I_{T H B}+R_{H B}+E_{H B D}+E_{T H B D}+I_{N H B D}+I_{T H B D}+R_{H B D}
\end{gathered}
$$

Susceptible individuals acquire HBV infection following effective contact with individuals infected with $\mathrm{HBV}$ (i.e. those in the $E_{H B}, I_{N H B}$ and $I_{T H B}$ classes) at a rate $\beta_{1}$, given by

$$
\beta_{1}=\frac{\theta_{B}\left(E_{H B}+\mu_{3} I_{N H B}+\mu_{4} I_{T H B}\right)}{N} ; N=S+E_{H B}+I_{N H B}+I_{T H B}
$$

where $\theta_{B}$ is the effective contact rate for HBV transmission. Further, the modification parameters $\mu_{3}=1$ and $\mu_{4}<1$ account for the relative infectiousness of individuals in the $I_{N H B}$ and $I_{T H B}$ classes in comparison to those in the $E_{H B}$ class. That is individuals in $I_{N H B}$ are more infectious than those individuals in the $E_{H B}$ class, likewise, $I_{T H B}$ are less infectious than those in the $I_{N H B}$ class (because the use of treatment significantly reduces the viral load in those being treated).

Similarly, individuals in the susceptible classes $\left(E_{H B}, I_{N H B}\right.$ and $\left.I_{T H B}\right)$ acquire $\mathrm{HBD}$, following effective contact with individuals infected with HBD

(i.e., those in the $E_{H B D}, I_{N H B D}$ and $I_{T H B D}$ ) at a rate $\beta_{2}$, given by

$$
\beta_{2}=\frac{\theta_{B D}\left(E_{H B D}+\mu_{5} I_{N H B D}+\mu_{6} I_{T H B D}\right)}{N} ; N=S+E_{H B D}+I_{N H B D}+I_{T H B D}
$$

where $\theta_{B D}$ is the effective contact rate for HBD transmission. Further, the modification parameters $\mu_{5}=1$ and $\mu_{6}<1$ account for the relative infectiousness of individuals in the $I_{N H B D}$ and $I_{T H B D}$ classes in comparison to those in the $E_{H B D}$ class. That is, individuals in the $I_{N H B D}$ class are more infectious than those in the $E_{H B D}$ class, and likewise, $I_{T H B D}$ are less infectious than those in $I_{N H B D}$ class (because the use of treatment significantly reduces the viral load in those being treated)

\section{Invariant Region}

The HBV-HDV co-infection model will be analyzed in a validity region in order to show that it is biologically meaningful and the region is feasible for human population. We assume that all the state variables and parameters are positive all the time, $t \geq 0$. We shall prove that the HBV-HDV co-infection model is well-posed by proving the boundedness and positivity of the solutions of the model with the non-negative initial solution for all time. We will obtain the region by considering the following theorems;

Theorem 3.1 (Boundedness). The solution set

$$
\left\{S(t), E_{H B}(t), E_{T H B}(t), I_{H B}(t), I_{N H B}(t), I_{T H B}(t), R_{H B}(t), E_{H B D}(t), E_{T H B D}(t), I_{H B D}(t), I_{N H B D}(t), I_{T H B D}(t), R_{H B D}(t)\right\}
$$

is contained and bounded in the feasible region $D$.

Proof. The total human population can be determined by

$$
N(t)=S(t)+E_{H B}(t)+E_{T H B}(t)+I_{N H B}(t)+I_{T H B}(t)+R_{H B}(t)+E_{H B D}(t)+E_{T H B D}(t)+I_{N H B D}(t)+I_{T H B D}(t)+R_{H B D}(t)
$$

Now adding the right hand side of equations (2.1) - (2.13)

So, the time derivatives, $\frac{d N}{d t}$, along solutions of system is obtained as

$$
\begin{aligned}
\frac{d N}{d t}=\pi & -\mu S-\mu E_{H B}-\mu E_{T H B}-\mu I_{N H B}-\mu I_{T H B}-\mu R_{H B}-\mu E_{H B D}-\mu E_{T H B D}-\mu I_{N H B D}-\mu I_{T H B D}-\mu R_{H B D}-\delta I_{T H B} \\
& -\delta_{1} I_{N H B}-\delta_{2} E_{T H B D}-\delta_{3} I_{T H B D}-\delta_{4} I_{N H B D} \leq \pi-\mu N .
\end{aligned}
$$

That is, $\frac{d N}{d t}=\pi-\mu N-\delta I_{T H B}-\delta_{1} I_{N H B}-\delta_{2} E_{T H B D}-\delta_{3} I_{T H B D}-\delta_{4} I_{N H B D} \leq \pi-\mu N$ Assume that the initial condition for model satisfies $N(0) \leq \frac{\pi}{\mu}$, where

$$
N(0)=S(0)+E_{H B}(0)+E_{T H B}(0)+I_{N H B}(0)+I_{T H B}(0)+R_{H B}(0)+E_{H B D}(0)+E_{T H B D}(0)+I_{N H B D}(0)+I_{T H B D}(0)+R_{H B D}(0)
$$

Then, applying the Gronwall's inequality gives

$N(t) \leq \frac{\pi}{\mu}+\left(N(0)-\frac{\pi}{\mu}\right) e^{-\mu t}$ whenever $(0) \leq \frac{\pi}{\mu}$.

So, taking the limit as $t \rightarrow \infty$ yields $(t) \leq \frac{\pi}{\mu}$. This shows that the feasible region for the model exists and is bounded by $N(t) \leq \frac{\pi}{\mu}$. It means that all the solutions of system are nonnegative in $D$ for any time $t>0$ and this represents human population. 


\subsection{Positivity of solutions}

Lemma1: Let the set of initial solution be

$$
\left\{\left(S(0), E_{H B}(0), E_{T H B}(0), I_{H B}(0), I_{N H B}(0), I_{T H B}(0), R_{H B}(0), E_{H B D}(0), E_{T H B D}(0), I_{H B D}(0), I_{N H B D}(0), I_{T H B D}(0), R_{H B D}(0)\right) \in \Phi\right\} \text {. }
$$

Then, the solution set

$$
\left\{S(t), E_{H B}(t), E_{T H B}(t), I_{H B}(t), I_{N H B}(t), I_{T H B}(t), R_{H B}(t), E_{H B D}(t), E_{T H B D}(t), I_{H B D}(t), I_{N H B D}(t), I_{T H B D}(t), R_{H B D}(t)\right\}
$$

of the HBV-HDV co-infection transmission model is non-negative for $t>0$.

Proof. Assume that the set of initial solutions,

$$
\left\{\left(S(0), E_{H B}(0), E_{T H B}(0), I_{H B}(0), I_{N H B}(0), I_{T H B}(0), R_{H B}(0), E_{H B D}(0), E_{T H B D}(0), I_{H B D}(0), I_{N H B D}(0), I_{T H B D}(0), R_{H B D}(0)\right) \geq 0\right\},
$$

then the first equation, that is equation (2.1) can be written as

$$
\frac{d S}{d t} \geq \pi-\left(\beta_{1} I_{H B}-\mu\right) S=\pi-\beta(t) S
$$

where $\beta(t)=\beta_{1} I_{H B}+\mu$.

Equation (3.1) is a linear first order ordinary differential equation in $S$ with the solution

$$
S(t)=S(0) \exp \left(\int_{0}^{t}-\beta(S) d s\right) \times \int_{0}^{t} \pi \exp \left(\int_{0}^{u} \beta(\omega) d \omega\right) \geq 0 .
$$

Hence, $S(t) \geq 0 \quad \forall \quad \mathrm{t} \geq 0$.

In a similar manner, the remaining state variables are obtained such that

$$
\begin{aligned}
& E_{H B}(t) \geq E_{H B}(0) \operatorname{Exp}(-(\mu+\tau+\omega)) \geq 0, \\
& E_{T H B}(t) \geq E_{T H B}(0) \operatorname{Exp}(-(\alpha+\mu)) \geq 0, \\
& I_{H B}(t) \geq I_{H B}(0) \operatorname{Exp}\left(-\left(\rho_{2}+\rho_{1}\right)\right) \geq 0, \\
& I_{N H B}(t) \geq I_{N H B}(0) \operatorname{Exp}\left(-\left(\mu+\delta_{1}\right)\right) \geq 0, \\
& I_{T H B}(t) \geq I_{T H B}(0) \operatorname{Exp}\left(-\left(\mu+\lambda_{1}+\lambda+\delta\right)\right) \geq 0, \\
& R_{H B}(t) \geq R_{H B}(0) \operatorname{Exp}\left(-\left(\mu+\phi_{1}\right)\right) \geq 0 . \\
& E_{H B D}(t) \geq E_{H B D}(0) \operatorname{Exp}(-(\mu+\kappa+\zeta)) \geq 0, \\
& E_{T H B D}(t) \geq E_{T H B D}(0) \operatorname{Exp}\left(-\left(\theta(1+c)+\mu+\varepsilon+\delta_{2}\right)\right) \geq 0, \\
& I_{H B D}(t) \geq I_{H B D}(0) \operatorname{Exp}\left(-\left(\varepsilon_{2}+\varepsilon_{1}\right)\right) \geq 0, \\
& I_{N H B D}(t) \geq I_{N H B D}(0) \operatorname{Exp}\left(-\left(\mu+\delta_{4}\right)\right) \geq 0, \\
& I_{T H B D}(t) \geq I_{T H B D}(0) \operatorname{Exp}\left(-\left(\mu+\eta+\delta_{3}\right)\right) \geq 0, \\
& R_{H B D}(t) \geq R_{H B D}(0) \operatorname{Exp}\left(-\left(\mu+\phi_{2}\right)\right) \geq 0 .
\end{aligned}
$$

This completes the proof of the lemma 1 .

Therefore, the biological validity of the HBV-HDV co-infection model is stated in lemma 2.

Lemma 2: The HBV-HDV co-infection model is well posed and valid in the set

$$
\begin{aligned}
D= & \left\{S(t), E_{H B}(t), E_{T H B}(t), I_{H B}(t), I_{N H B}(t), I_{T H B}(t), R_{H B}(t), E_{H B D}(t), E_{T H B D}(t), I_{H B D}(t), I_{N H B D}(t), I_{T H B D}(t), R_{H B D}(t) \in \mathbb{R}_{+}^{13}\right. \\
& \left.: N(t) \leq \frac{\pi}{\mu}\right\}
\end{aligned}
$$

According to [16], the HBV-HDV co-infection model is biologically meaningful and epidemiologically well posed in the region $D$. 


\begin{tabular}{|c|c|c|c|}
\hline $\mathbf{S} / \mathbf{N}$ & Parameter & Value & Reference \\
\hline 1 & $\mu$ & 0.021 & [17] and [18] \\
\hline 2 & $\pi$ & 1000 & Assumed \\
\hline 3 & $\beta_{1}$ & 0.37 & Assumed \\
\hline 4 & $\tau$ & 0.50 & [19] and [20] \\
\hline 5 & $\beta_{2}$ & 0.37 & Assumed \\
\hline 6 & $c_{1}$ & 0.08 & {$[17]$} \\
\hline 7 & $c_{2}$ & 0.08 & {$[17]$} \\
\hline 8 & $\psi$ & 0.047 & Assumed \\
\hline 9 & $\varphi \Rightarrow \Phi$ & 0.043 & Assumed \\
\hline 10 & $\lambda_{1}$ & 0.01 & Assumed \\
\hline 11 & $\lambda_{2}$ & 0.015 & [17] \\
\hline 12 & $\omega$ & 0.02 & Assumed \\
\hline 13 & $\alpha$ & 0.015 & [17] \\
\hline 14 & $\rho_{1}$ & 0.33 & [19] and [20] \\
\hline 15 & $\rho_{2}$ & 0.33 & [19] and [20] \\
\hline 16 & $\theta$ & 0.08 & {$[17]$} \\
\hline 17 & $c$ & 0.08 & {$[17]$} \\
\hline 18 & $\varepsilon_{1}$ & 0.038 & Assumed \\
\hline 19 & $\varepsilon_{2}$ & 0.038 & Assumed \\
\hline 20 & $\eta$ & 0.028 & Assumed \\
\hline 21 & $\mathrm{~K}$ & 0.50 & [19] and [20] \\
\hline 22 & $\zeta$ & 0.032 & Assumed \\
\hline 23 & $\epsilon$ & 0.035 & Assumed \\
\hline 24 & $\phi_{1}$ & 0.92 & Assumed \\
\hline 25 & $\phi_{2}$ & 0.92 & Assumed \\
\hline 26 & $\delta$ & 0.068 & {$[17]$} \\
\hline 27 & $\delta_{1}$ & 0.068 & {$[17]$} \\
\hline 28 & $\delta_{2}$ & 0.068 & {$[17]$} \\
\hline 29 & $\delta_{3}$ & 0.068 & {$[17]$} \\
\hline 30 & $\delta_{4}$ & 0.068 & [17] \\
\hline
\end{tabular}

Table 1: Parameters value for HBV-HDV co-infection

\section{Simulation Results and Discussion}

Here we investigates the impact of the various parameters of the model developed by [14] through simulation using MATLAB. This will help provide the best framework for control strategies of the HBV-HDV co-infection in a population. Table 1 shows the set of parameter values used in the simulation for HBV-HDV co-infection.

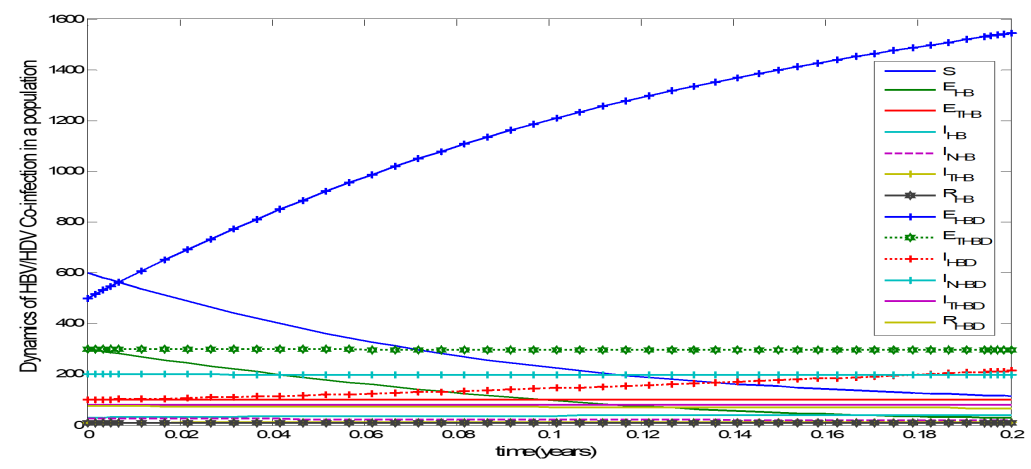

Figure 4.1: A graph showing the dynamics of HBV-HDV co-infection in a population.

Figure 4.1, shows all the equations describing the dynamics of HBV-HDV co-infection in a population. 

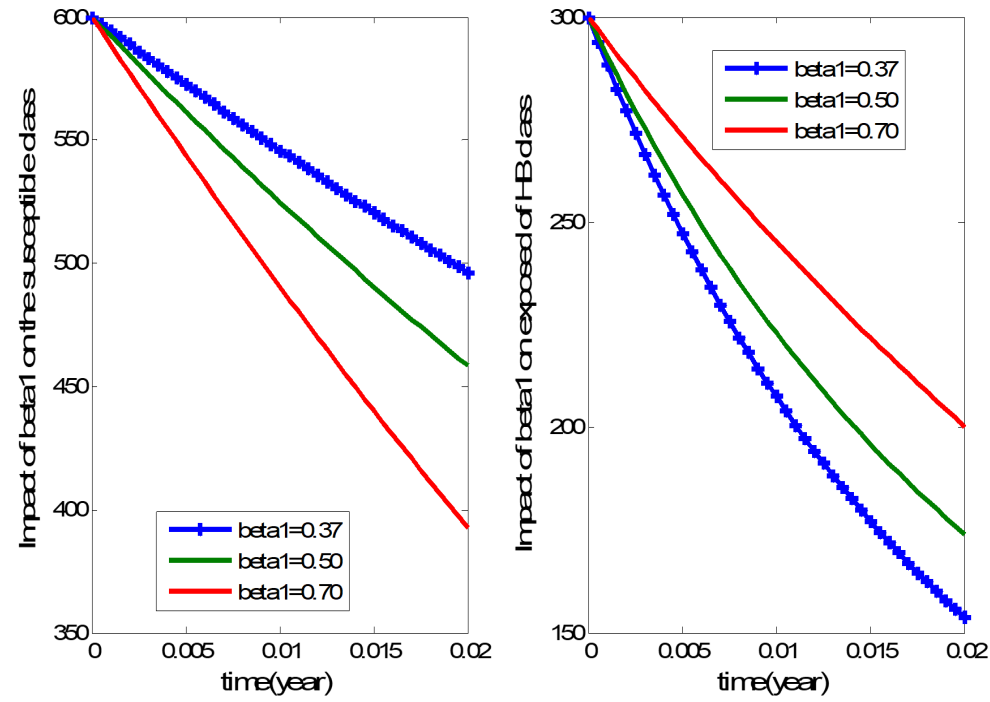

Figure 4.2: Graphs showing the impact of $\beta_{1}$ (contact rate for $I_{H B}$ with susceptible individuals) in the population.

Figure 4.2(a): The graph on the left, showing the impact of $\beta_{1}$ on the susceptible class.

Figure 4.2(b): The graph on the right, showing the impact of $\beta_{1}$ on exposed of HB class.

From figure 4.2(a), increasing $\beta_{1}$ decreases the number of susceptible people in the population. On the contrary, increasing $\beta_{1}$ increases the number of individuals in the population who are exposed to HB (see figure 4.2b). Therefore, as a control strategy, efforts should be geared towards ensuring that the contact rate is drastically reduced. This is achievable if more susceptible individuals are immunized against HBV infection. This is so because upon immunization the individual will lose his/her potency of contacting the disease for a considerable period of time. Active vaccination (Energix-B and Recombivax-HB) is recommended as this will offer long lasting active immunity against HBV and invariably will help control the spread of HBV in the population.
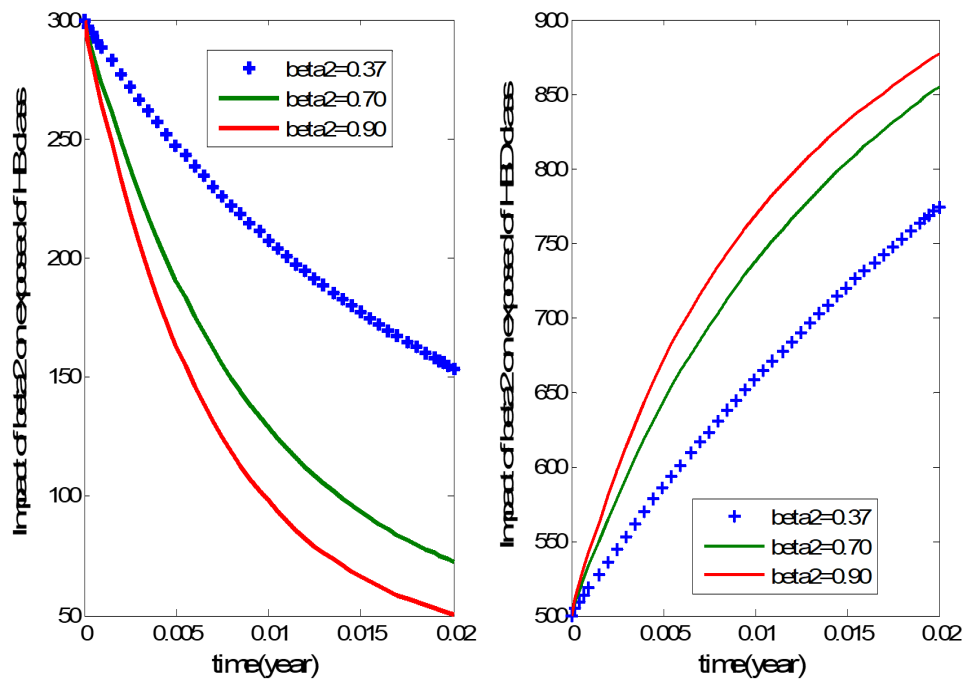

Figure 4.3: Graphs showing the impact of $\beta_{2}$ (contact rate for $I_{H B D}$ with susceptible classes) in the population.

Figure 4.3(a): The graph on the left, showing the impact of $\beta_{2}$ on exposed of HB class.

Figure 4.3(b): The graph on the right, showing the impact of $\beta_{2}$ on exposed of HBD class.

In figure 4.3(a), we observe that increasing $\beta_{2}$ brings about a decrease in the number of exposed of HB people in the population. On the contrary, increasing $\beta_{2}$ increases the number of individuals in the population who are exposed of HBD as can be clearly seen in figure 4.3(b) above. Therefore, as a control strategy, we must increase awareness of the disease amongst the people. Once people are aware of the disease, then they can indulge on other diligent preventive measures: vaccine, not sharing needles or other drug paraphernalia and not sharing items such as tooth brushes and razors. Individuals can also get vaccinated against HBV once they become aware. Therefore, efforts should be targeted at increasing the above mentioned strategies for effective control of HBV in the population. Once we are able to effectively reduce the number of HBV individuals in the population through the above mentioned control strategies, then we would have succeeded in reducing the contact rate of $\mathrm{HB}$ and exposed of $\mathrm{HBD}$ in the population. 

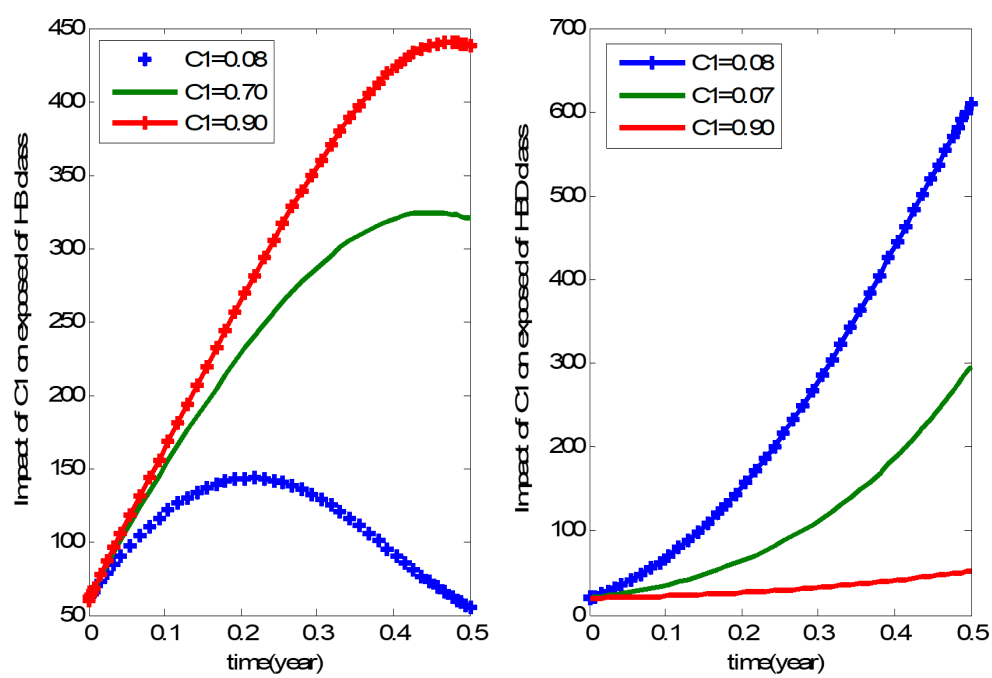

Figure 4.4: Graphs showing the impact of $c_{1}$ (awareness) in the population.

Figure 4.4(a): The graph on the left, showing the impact of $c_{1}$ on exposed of HB class.

Figure 4.4(b): The graph on the right, showing the impact of $c_{1}$ on exposed of HBD class.

In figure 4.4(a), increasing $c_{1}$ (awareness), shows an increase on the number of exposed of HB individuals in the population as compare to the number of exposed of HBD individuals in the population. Conversely, in figure 4.4(b), increasing $c_{1}$ shows a drastic decrease on the number of exposed HBD people in the population in contrast to HBV individuals in the population. From this we can see that awareness is a very good control measure, this is so because when awareness increased more people will remain in exposed of $\mathrm{HB}$ class which is better and much easier to treat than exposed of HBD patients. Therefore, to effectively control HBV-HDV co-infection in any given population, we must increase awareness of the HBV disease amongst the people. Once people are aware of the disease, then they can indulge on other diligent preventive measures: condom use, not sharing needles or other drug paraphernalia, not sharing items such as tooth brushes and razors. Individuals can also get vaccinated against HBV once they become aware. Therefore, efforts should be targeted at increasing the above mentioned strategies for effective control of HBV - HDV co-infection in the population.
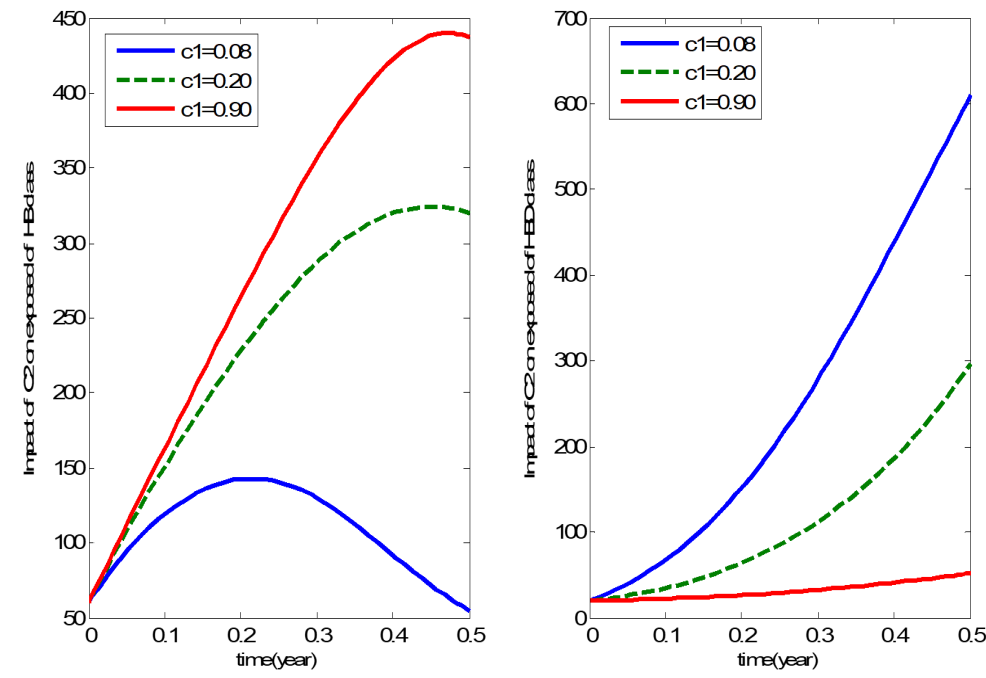

Figure 4.5: Graphs showing the impact of $c_{2}$ (vaccine) in the population.

Figure 4.5(a): The graph on the left, showing the impact of $c_{2}$ on exposed of HB class.

Figure 4.5(b): The graph on the right, showing the impact of $c_{2}$ on exposed of HBD class.

Note: Vaccine is to prevent people from contracting the infection while therapy is used to cure those infected with the disease.

Again we see in figure 4.5(a), increasing $c_{2}$ (vaccine), shows a great increase on the number of exposed of HB individuals in the population compare to the number of HBD individuals in the population. Subsequently, in figure 4.5(b), increasing $c_{2}$ shows a drastic decrease on the number of exposed HBD people in the population compare to the number of HBV individuals in the population. From this we can see that vaccine is a very good control measure, this is so because when more people are vaccinated even if they are already exposed to HB, their condition will never deteriorate to exposed of HBD (that is HBV-HDV co-infection) which always has a worst outcome than exposed of HBV or even infectious of HBV. Therefore, to effectively control HBV-HDV co-infection in any given population, the need for people to be 
vaccinated against HBV is highly recommended as this will go a long way in helping to reduce HBV-HDV co-infection. Active vaccination (Energix-B and Recombivax-HB) is recommended; this will offer long lasting active immunity against HBV and invariably will help control HBV-HDV co-infection in the population.
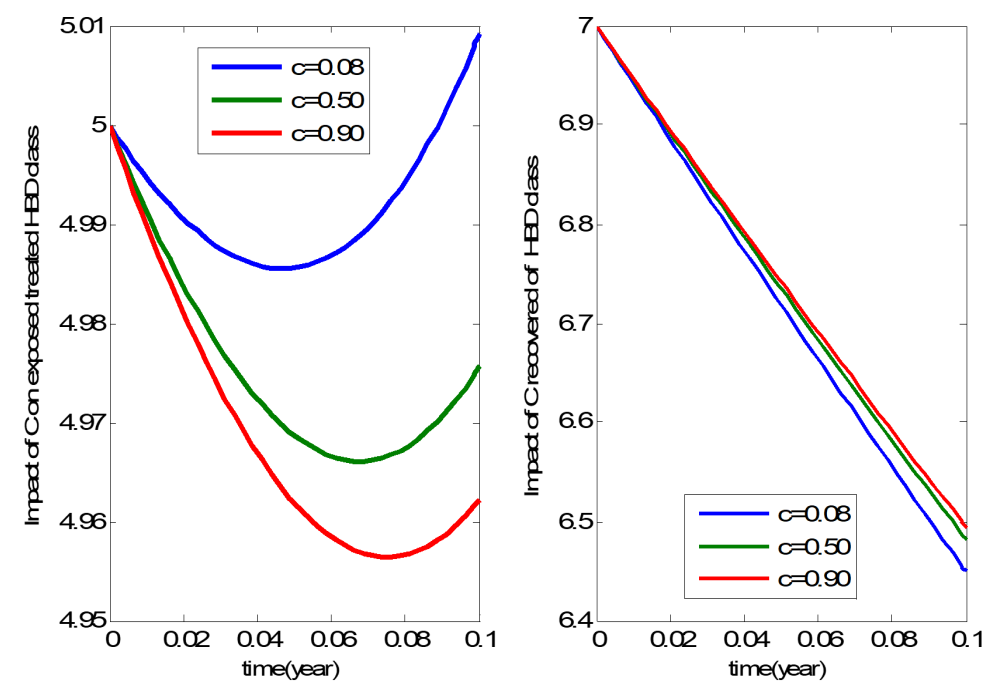

Figure 4.6: Graphs showing the impact of $c$ (therapy) in the population.

Figure 4.6(a): The graph on the left, showing the impact of $c$ on exposed treated HBD class.

Figure 4.6(b): The graph on the right, showing the impact of $c$ on recovered of HBD class.

Note: Vaccine is used as a preventive measure while therapy is used to cure those infected with the disease already.

From figure 4.6(a), the impact of $c$ (therapy) as a control measure is noticeable. If $c$ (therapy) increases, then there is a noticeable decrease on the number of exposed and treated individuals in the population. Similarly, in figure 4.6(b), we can see an increase on the number of recovered HBD individual in the population upon the increase of $c$ (therapy). This implies that therapy has a positive impact as control measure in the population. More exposed treated HBD individuals will recover after using the drug. The available drugs for the treatment of HBV which also should be used for patients exposed of HBD are: (1) Lamivudine (LMV) or Ribavirine (2) Eggylated Alpa-interferon (IFN). However, it is worthy to note; (i) a large doses of Alpha-interferon may be given for up to 12 months (ii) Alpha-interferon can cause the disease to go into remission (according to World Health Organisation).
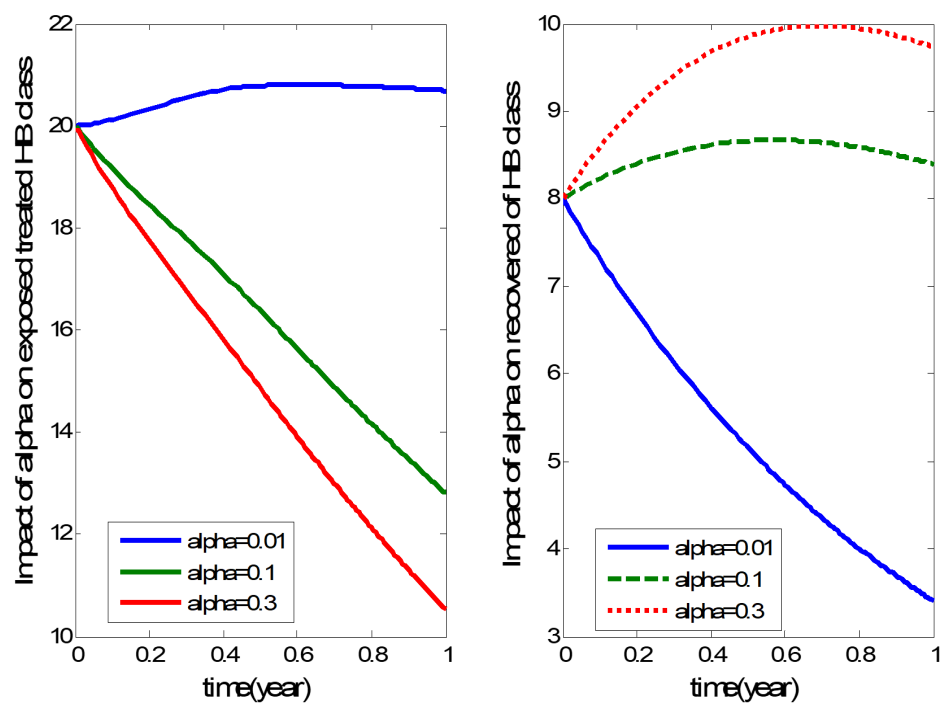

Figure 4.7: Graphs showing the impact of $\alpha$ (the rate at which individuals who are exposed and being treated of HBV recover) in the population.

Figure 4.7(a): The graph on the left, showing the impact of $\alpha$ on exposed treated HB class.

Figure 4.7(b): The graph on the right, showing the impact of $\alpha$ on recovered of HB class.

In figure 4.7(a), we can clearly see that increasing alpha shows a reduction on the number of exposed treated individuals in the population. In figure 4.7(b), increasing alpha shows an increase on the number of people who recovered from HB in the population. As a control measure, people who are diagnosed of being exposed to HBV should be encouraged to go for treatment. From the graphs above, more than $80 \%$ of exposed of HBV individuals who are treated of HBV recovered. 

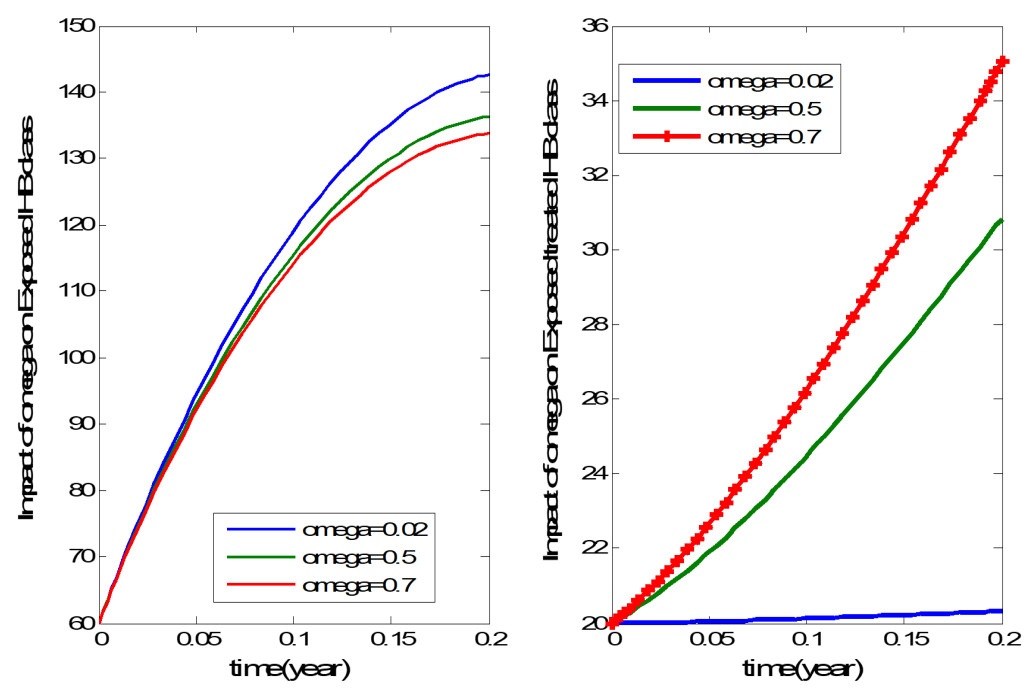

Figure 4.8: Graphs showing the impact of $\omega$ (the rate at which individuals who are exposed to HBV enter into exposed and treated compartment) in the population.

Figure 4.8(a): The graph on the left, showing the impact of $\omega$ on exposed HB class.

Figure 4.8(b): The graph on the right, showing the impact of $\omega$ on exposed treated HBD class. Graph in figure 4.8(a) shows a gradual decrease on the number of exposed HB individuals in the population as the value of omega was increasing. In figure 4.8(b), an increase in omega shows a great increase on the number of exposed treated of HB individuals in the population. The reason for the increase on the number of treated people in the population could be as a result of awareness. When more people are aware of the disease and go for screening, the infected ones will start treatment; this will eventually lead to recovery. Often time, people may not know that they are exposed of HBV until irreversible damage is done to the liver before they will become aware of the infection so as a control strategy, awareness is strongly recommended.
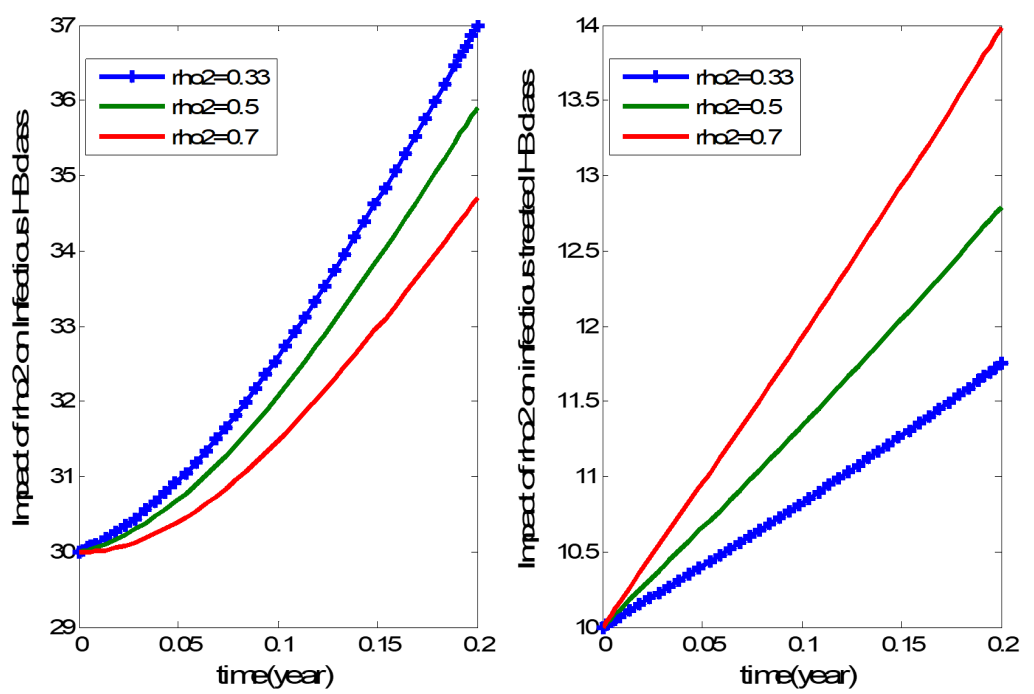

Figure 4.9: Graphs showing the impact of $\rho_{2}$ in the population.

Figure 4.9(a): The graph on the left, showing the impact of $\rho_{2}$ on infectious HB class.

Figure 4.9(b): The graph on the right, showing the impact of $\rho_{2}$ on infectious treated HB class.

From figure 4.9(a), increasing $\rho_{2}$ shows a decrease in the number of infectious HB individuals in the population. Thus, an increase in $\rho_{2}$ shows a very high increase on the number of infectious treated HB people in the population (see figure $4.9 \mathrm{~b}$ ). From the above graphs, it is obvious that the more people who are infected with HBV go for treatment the less the number of HBV infected individuals in the population. Therefore, as a control strategy, people who are diagnosed of HBV should be encouraged to go for treatment. Government should also help by providing free medical care for HBV infected persons. Also non-governmental organizations should even help in sponsoring medical treatment for HBV patients. Therefore, as a control measure, HBV infected individuals should be made to go for treatment this will help reduce $\mathrm{HBV}$ in a population and invariably reduce HBV-HDV co-infection in the population. 

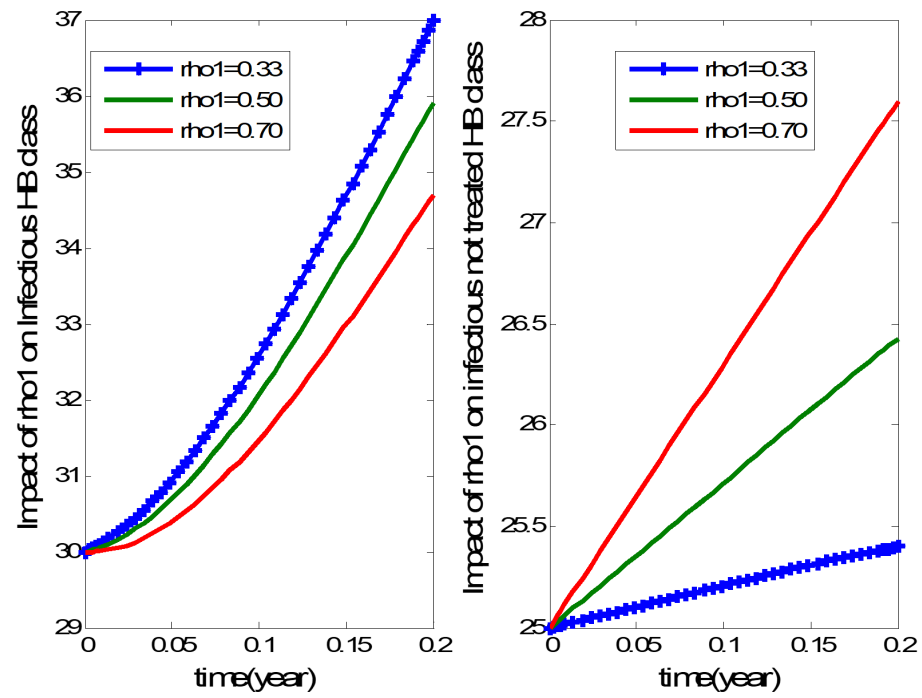

Figure 4.10: Graphs showing the impact of $\rho_{1}$ (the rate at which individuals who are infectious of HBV enter into infectious and not being treated of HBV class) in the population.

Figure 4.10(a): The graph on the left, showing the impact of $\rho_{1}$ on infectious HB class.

Figure 4.10(b): The graph on the right, showing the impact of $\rho_{1}$ on infectious not treated HB class.

From figure 4.10(a), increasing $\rho_{1}$ reduces the number of infectious of HB individuals in the population. Also in figure 4.10(b), increasing $\rho_{1}$ increases the number of infectious not treated HB individuals in the population. Actually, $\rho_{1}$ has a very negative impact or effect in the population. People who are infectious of HBV should be encouraged to go for treatment as recommended earlier. For no reason should people who are diagnosed of HBV stay without going for treatment. Therefore, $\rho_{1}$ is not contributing in any way to the control of HBV and HBV/HDV co-infection in the population, concerted effort should be made to avoided this ugly scenario.
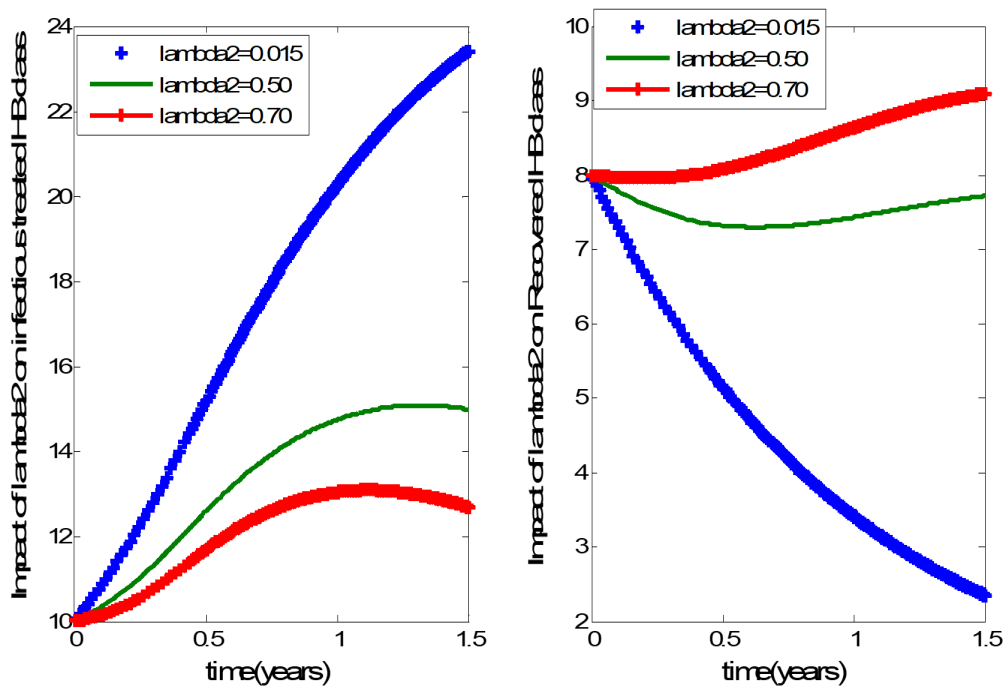

Figure 4.11: Graphs showing the impact of $\lambda_{2}$ (the rate at which individuals who are infectious and being treated of HBV recover from HBV) in the population.

Figure 4.11(a): The graph on the left, showing the impact of $\lambda_{2}$ on infectious treated HB class.

Figure 4.11(b): The graph on the right, showing the impact of $\lambda_{2}$ on recovered HB class.

From figure 4.11(a) above, if $\lambda_{2}$ increases then the number of infectious treated HB people decreases in the population. On the other hand, in figure 4.11(b), increasing $\lambda_{2}$ also increases the number of recovered of HB individuals in the population. This implies that treatment of HBV infected people has a positive impact in the population as more infected individuals will recover after treatment as shown in the graph (figure $4.11 \mathrm{~b}$ ) above. If the infection of HBV is acute, then enough rest is highly recommended and also care should be taken to treat the exhibited symptoms. But on the other hand, if the infection of HBV is chronic, then the use of HBV drugs is recommended. The available drugs for the treatment of HBV are: (1) Lamivudine (LMV) or Ribavirine (2) Eggylated Alpa-interferon (IFN). If more infected HBV people are treated, it will help control HBV infection in the population and in turn control HBV-HDV co-infection. The recovered individuals can even get immunized to avoid contracting it again.

Figure 4.12(a): The graph on the left, showing the impact of $\lambda_{1}$ on exposed HB class.

Figure 4.12(b): The graph on the right, showing the impact of $\lambda_{1}$ on infectious treated HB class. 

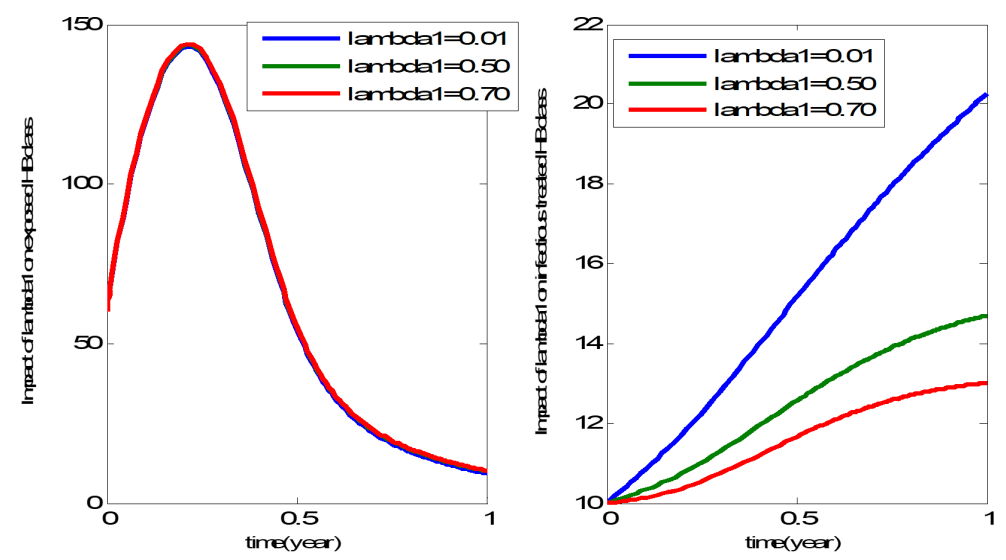

Figure 4.12: Graphs showing the impact of $\lambda_{1}$ (the rate at which individuals that are infectious and being treated of HBV goes back to exposed HBV class) in the population.

In figure 4.12(a) above, we will observe that increasing the value of $\lambda_{1}$ does not show any noticeable increase or decrease on the exposed $\mathrm{HB}$ class. On the contrary, in figure 4.12(b), it can be clearly seen that increasing $\lambda_{1}$ decreases the number of infectious treated HB individuals in the population. The condition such as shown here occurs when the disease (HBV) goes into remission in the body of the HBV infected person who is receiving treatment for HBV. When this happens, the patient will not exhibit any symptom(s) of HBV again, and will conclude that he or she has recovered from the infection. From the graphs above, we can see that this situation will arise when more people are infected of HBV and are being treated, some will recover fully and move to recovered class while very few(unnoticeable number) will go back again to exposed class (that is, the disease goes into remission in their body without their knowledge). This has a negative impact in the population and should be avoided. Therefore, as a control strategy, we recommend that the blood of HBV patients who is receiving treatment be screened thoroughly before they are certified recovered from HBV infection. This will help control HBV-HDV co-infection and or HBV-HDV super-infection in the population.
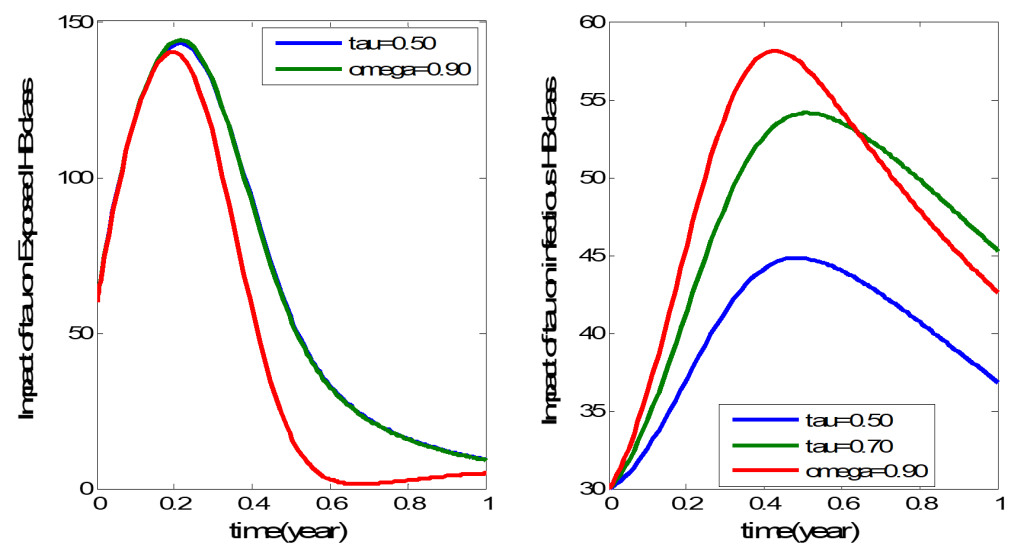

Figure 4.13: Graphs showing the impact of $\tau$ (the rate at which individuals who are exposed to HBV become infectious of HBV) in the population.

Figure 4.13(a): The graph on the left, showing the impact of $\tau$ on exposed HB class.

Figure 4.13(b): The graph on the right, showing the impact of $\tau$ on infectious HB class.

From figure 4.13(a), increasing $\tau$ decreases the number of individuals who are exposed of HBV in the population. On the contrary, increasing $\tau$ increases the number of infectious individual in the population (see figure $4.13 \mathrm{~b}$ ). Therefore, efforts should be made through awareness for people to know their HBV status. This if done will help individuals who are exposed of HBV to discover on time and go for treatment so that the person will not migrate to infectious stage. Therefore, as a control strategy, we recommend awareness and that people should have a blood test for the antibody to hepatitis B surface antigen. Early dictation of HBV is an advantage in curing the disease than when it has reached an advanced stage, often time the advance stage would have caused Fibrosis (scarring) and Cirrhosis (hardening of the liver). These conditions are too hard to reverse. This explains why persons diagnosed of HBV that has reached advanced stage often do not survive because of the irreversible damage already done to the liver as a result of the unawareness of the disease. 

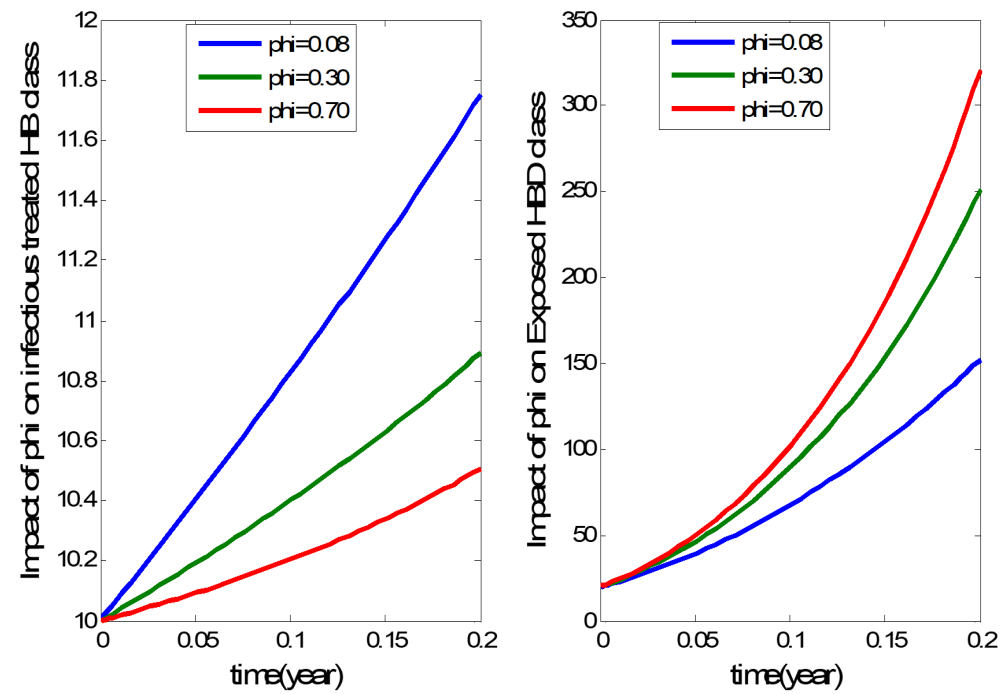

Figure 4.14: Graphs showing the impact of $\varphi$ (the rate at which individuals that are infectious and being treated of HBV become exposed to HDV) in the population.

Figure 4.14(a): The graph on the left, showing the impact of $\varphi$ (phi) on infectious treated HB class.

Figure 4.14(b): The graph on the right, showing the impact of $\varphi$ (phi) on exposed HBD class.

From figure 4.14(a), increasing phi decreases the numbers of infectious treated HB individuals in the population compare to the number of exposed HBD in the population. Also we notice an increase in the number of exposed of HBD people in the population by increasing phi compare to the number of infectious treated HB individuals in the population (see figure 4.14b). This condition has a negative impact on the control of $\mathrm{HBV}$ and the resulting consequence is that more people in the population will become exposed to HBD. As a control measure, adequate care should be taken to ensure that HB infectious people who are being treated do not contact HDV, for this will worsen their condition.
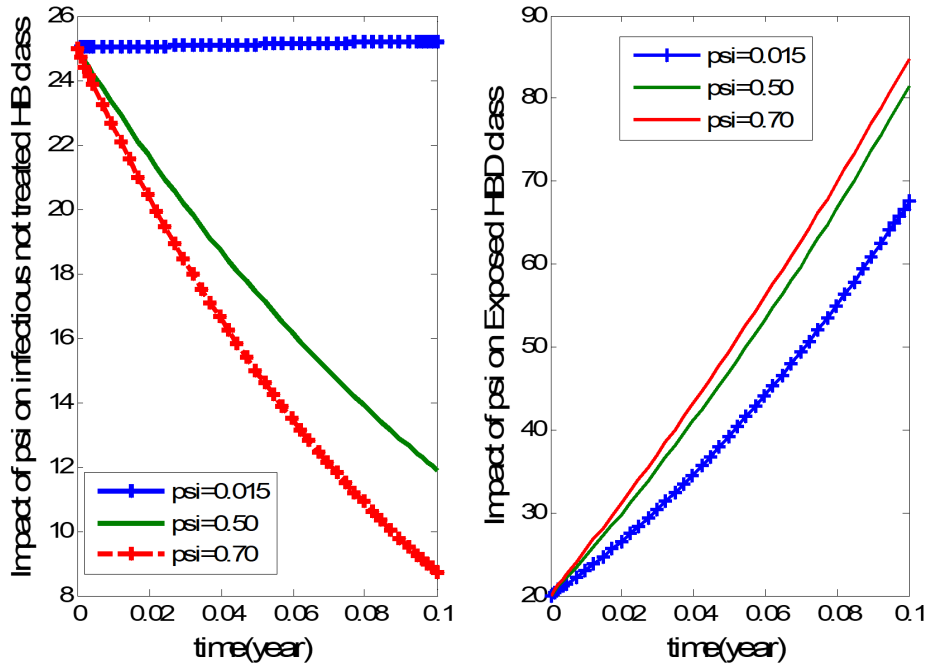

Figure 4.15: Graphs showing the impact of $\psi$ (the rate at which individuals who are infectious of HBV and not being treated become exposed to HDV ) in the population.

Figure 4.15(a): The graph on the left, showing the impact of $\psi$ (psi) on infectious not treated HB class.

Figure 4.15(b): The graph on the right, showing the impact of $\psi$ (psi) on exposed HBD class.

In figure 4.15(a), increasing $\psi$ shows a very high decrease on the number of infectious not treated HB individuals in the population. Also in figure 4.15(b), we notice a small increase in the number of exposed of HBD people in the population. This condition again has a negative impact on the control of HBV; hence the resulting consequence is that more people in the population will become exposed to HBD. As a control measure, HB infectious people should be given treatment. 

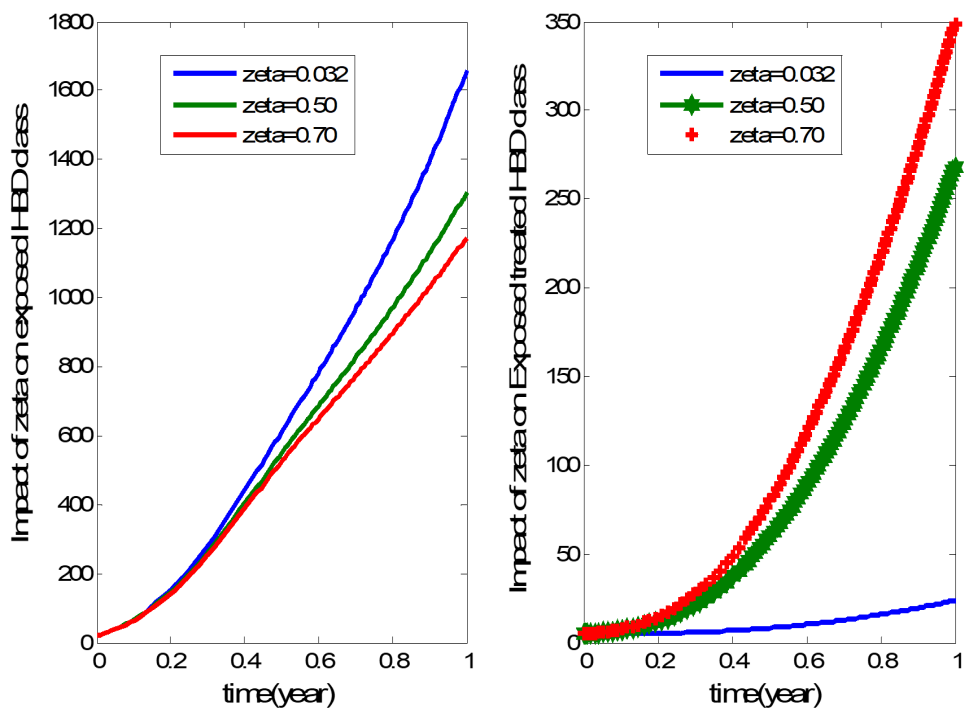

Figure 4.16: Graphs showing the impact of $\zeta$ (the rate at which HBV infectious individuals who are exposed to HDV enter into exposed HDV being treated class) in the population.

Figure 4.16(a): The graph on the left, showing the impact of $\zeta$ (zeta) on exposed HBD class.

Figure 4.16(b): The graph on the right, showing the impact of $\zeta$ (zeta) on exposed treated HBD class.

Graph in figure 4.16(a) shows a gradual decrease on the number of exposed of HBD individuals in the population as omega values increases. In figure 4.16(b), an increase in omega shows a great increase on the number of exposed treated of HBD individuals in the population. The reason for the increase on the number of treated people in the population could be as a result of awareness. When more people are aware of the disease and go for screening, the infected ones will start treatment; this will eventually lead to recovery. Often time, people may not know that they are exposed of HBD until irreversible damage is done to the liver before they will become aware of the infection so as a control strategy, awareness is strongly recommended.
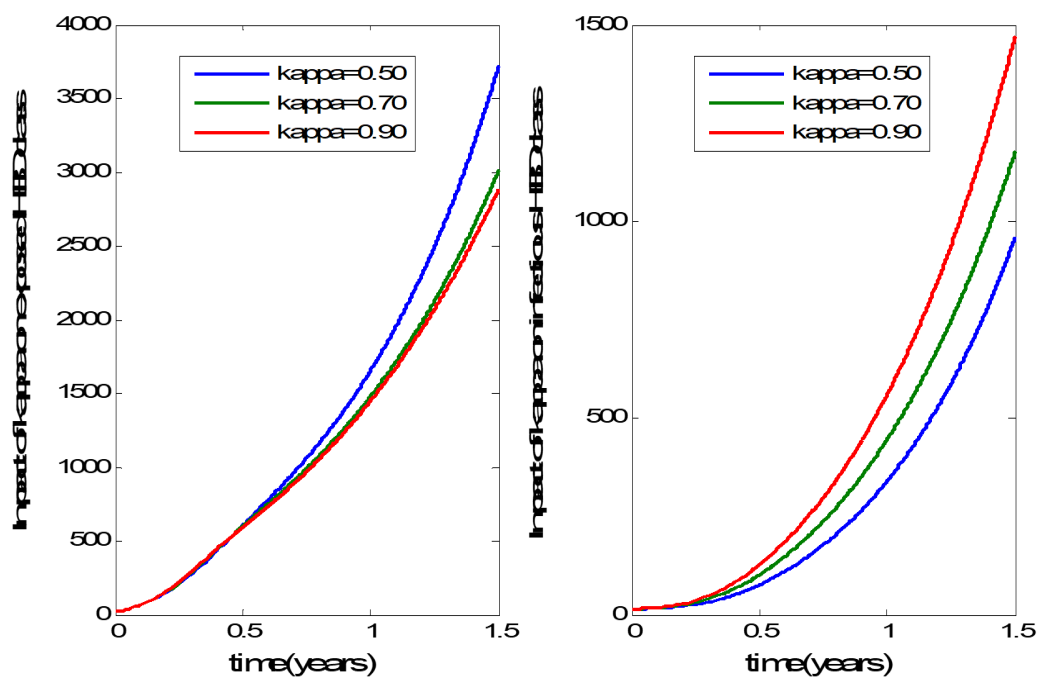

Figure 4.17: Graphs showing the impact of $\kappa$ (the rate at which HBV infectious individuals who are exposed to HDV become infectious of HBV-HDV ) in the population.

Figure 4.17(a): The graph on the left, showing the impact of $\kappa$ (kappa) on exposed HBD class.

Figure 4.17(b): The graph on the right, showing the impact of $\kappa$ (kappa) on infectious HBD class.

From figure 4.17(a), increasing $\kappa$ decreases the number of individuals who are exposed of HBD in the population. Conversely, increasing $\kappa$ increases the number of infectious of HBD individual in the population (see figure 4.17b). Therefore, intensive effort should be made by the government through awareness so that people will become aware and have their blood tested for the antibody to hepatitis B surface antigen. Hepatitis B surface antigen (HBsAg) is a platform for HDV to replicate and cause infection in the body, therefore early dictation of HBV and or HDV is an advantage in curing the disease than when it has reached an advanced stage, often time the advance stage would have caused Fibrosis (scarring) and Cirrhosis (hardening of the liver). These conditions are too hard to reverse. This explains why persons diagnosed of HBV that has reached advanced stage often do not survive because of the irreversible damage already done to the liver as a result of unawareness of the disease(s). The above measure if followed will help control HBV-HDV co-infection in a population. 

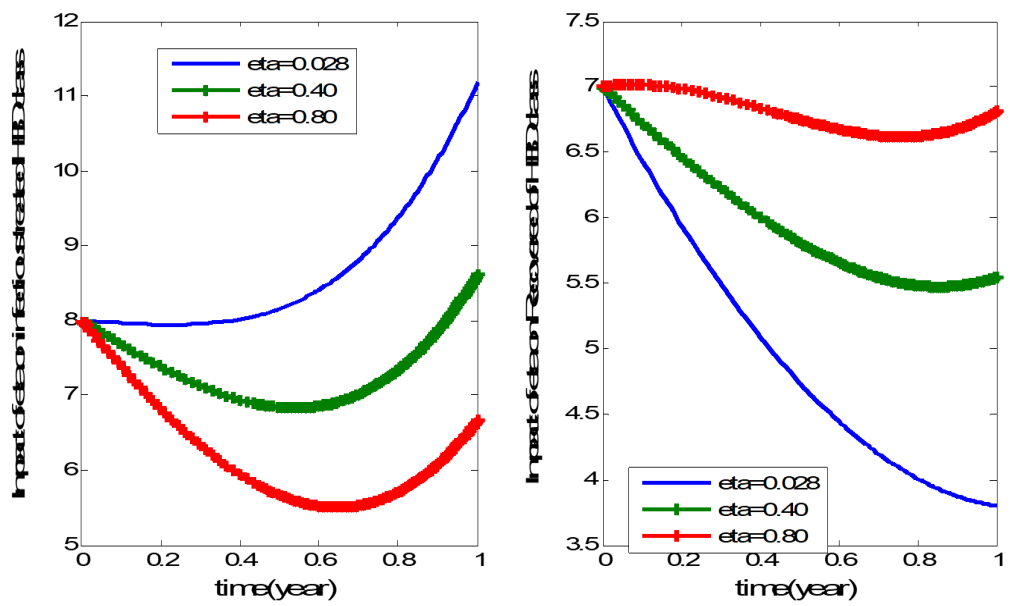

Figure 4.18: Graphs showing the impact of $\eta$ (the rate at which individuals that are infectious of HBV-HDV and are being treated recover in the population.

Figure 4.18(a): The graph on the left, showing the impact of $\eta$ (eta) on infectious treated HBD class.

Figure 4.18(b): The graph on the right, showing the impact of $\eta$ (eta) on recovered of HBD class.

From figure 4.18(a) above, if $\eta$ increases then the number of infectious treated HBD people decreases in the population. On the other hand, in figure 4.18(b), increasing $\eta$ also increases the number of recovered of HBD individuals in the population. This implies that treatment of HBD infected people has a positive impact in the population as more infected individuals will recover after treatment as shown in the graph (figure 4.18b) above. Worthy to note are: (i) large doses of Alpha-interferon may be given for up to 12 months for HDV patients (ii) Alpha-interferon can cause the disease (HDV) to go into remission (according to World Health Organisation) (iii) after treatment , people with HDV can still test positive for the condition. Therefore, the best control strategy for HDV and HBV-HDV co-infection in a population is to avoid exposure to HBV (that is, to effectively control HBV in the population).
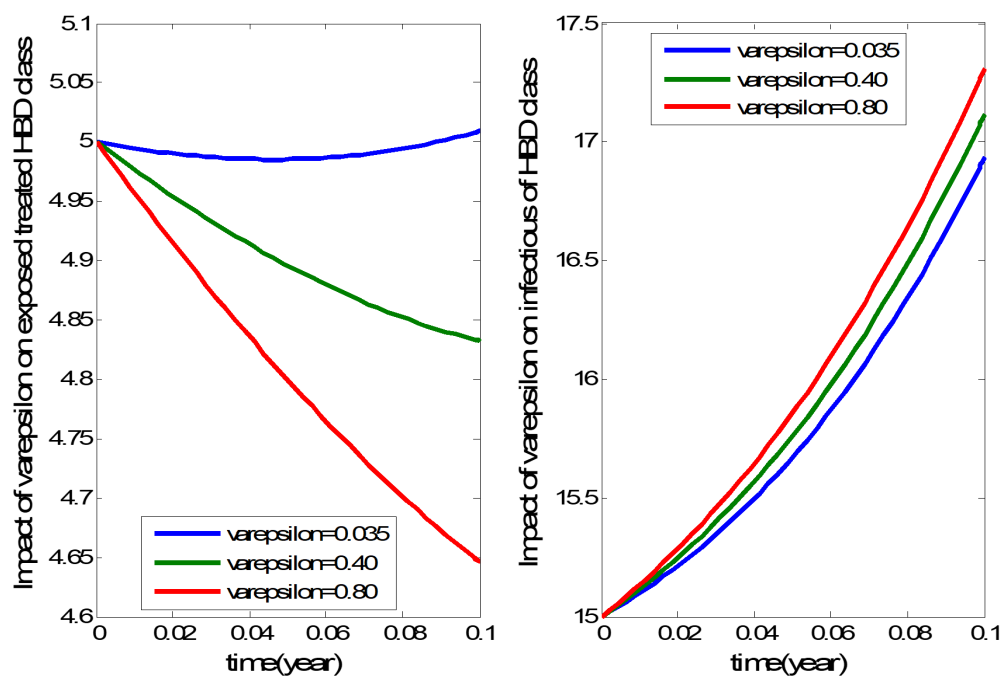

Figure 4.19: Graphs showing the impact of $\varepsilon$ (the rate at which HBV infectious individuals who are exposed to HDV and then being treated become infectious of HBV-HDV) in the population.

Figure 4.19(a): The graph on the left shows the impact of $\varepsilon$ (epsilon variant) on exposed treated HBD class.

Figure 4.19(b): The graph on the right shows the impact of $\varepsilon$ (epsilon variant) on infectious of HBD class.

From figure 4.19(a), increasing epsilon variant decreases the number of exposed treated HBD individuals in the population. Also we notice an increase in the number of infectious of HBD people in the population by increasing epsilon variant (see figure 4.19b). This condition has a negative impact on the control of HBD in the population. As a control measure, adequate care should be taken to ensure that HBD exposed treated people are faithful to their drugs, this will go a long way in helping them recover completely instead of deteriorating to infectious of HBD class . 

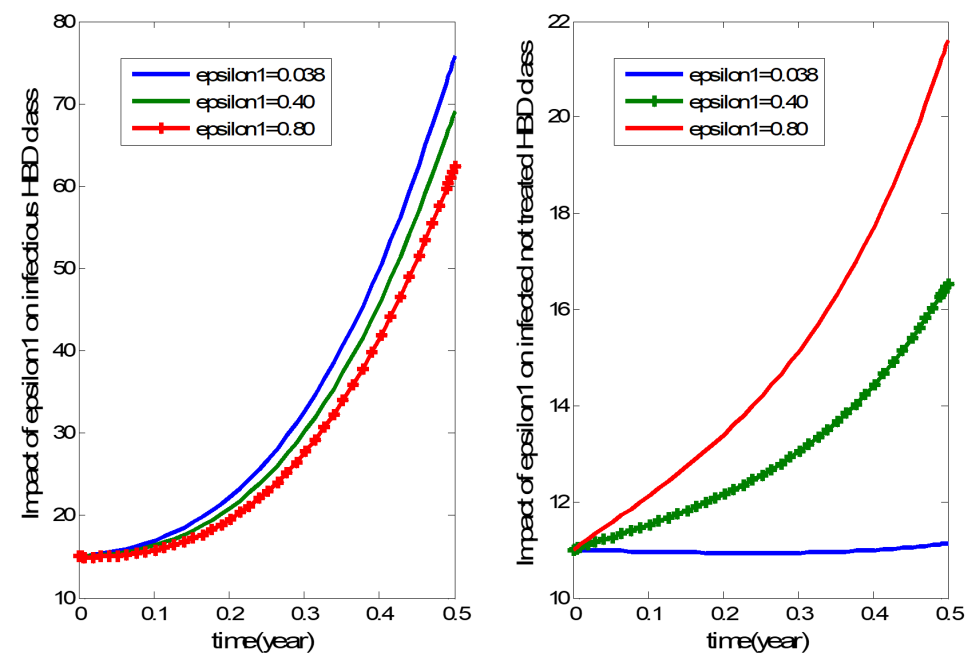

Figure 4.20: Graphs showing the impact of $\varepsilon_{1}$ (the rate at which individuals who are infectious of HBV-HDV enter into infectious HBV-HDV not being treated class) in the population.

Figure 4.20(a): Graph on the left, showing the impact of $\varepsilon_{1}$ (epsilon 1) on infectious of HBD class.

Figure 4.20(b): Graph on the right, shows the impact of $\varepsilon_{1}$ (epsilon 1) on infectious not treated HBD class.

From figure 4.20(a), increasing $\varepsilon_{1}$ reduces the number of infectious HBD individuals in the population. Also in figure 4.20(b), increasing $\varepsilon_{1}$ increases the number of infectious not treated HBD individuals in the population. Actually, $\varepsilon_{1}$ has a very negative impact or effect in the population. People who are infectious of HBD should be encouraged to go for treatment as recommended earlier. For no reason should people who are diagnosed of HBD be allowed to go away without receiving treatment. Therefore, $\varepsilon_{1}$ is not contributing in any way to the control of HBV in the population, this should be avoided.
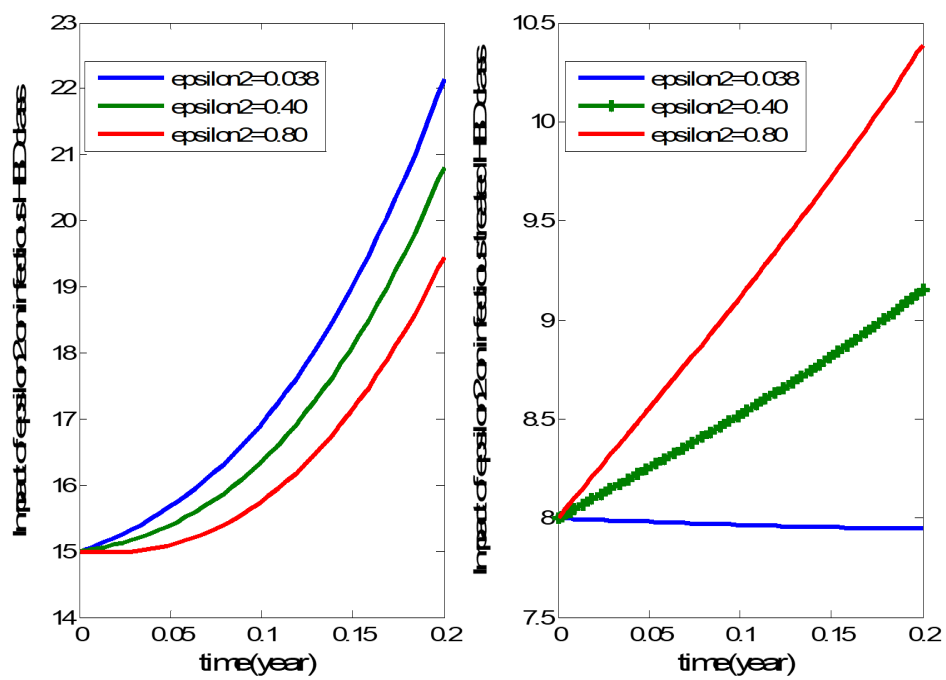

Figure 4.21: Graphs showing the impact of $\varepsilon_{2}$ (the rate at which individuals who are infectious of HBV-HDV enter into infectious HBV-HDV being treated class) in the population.

Figure 4.21(a): Graph on the left, showing the impact of $\varepsilon_{2}$ (epsilon 2) on infectious of HBD class.

Figure 4.21(b): Graph on the right, shows the impact of $\varepsilon_{2}$ (epsilon 2) on infectious treated HBD class.

From figure 4.21(a), increasing $\varepsilon_{2}$ shows a decrease in the number of infectious of HBD individuals in the population. Conversely, an increase in $\varepsilon_{2}$ shows a very high increase on the number of infectious treated HBD people in the population (see figure 4.21b). From the above graphs, it is obvious that the more people who are infected with HBD go for treatment the less the number of HBD infected individuals in the population. Therefore, as a control strategy, people who are diagnosed of HBD should be encouraged to go for treatment. Government should also help by providing free medical care for HBD infected persons. Also non-governmental organizations should even help in sponsoring medical treatment for HBD patients. Therefore, as a control measure, HBD infected individuals should be made to go for treatment this will help reduce HBV-HDV co-infection in the population. 


\section{Conclusion}

We investigates the impact of the various parameters of the mathematical model for Hepatitis B virus - Hepatitis D virus (HBV - HDV) co-infection with controls (awareness, vaccine and therapy). Furthermore, simulations are carried out on the equations of the model using MATHLAB and the results are discussed. From the results of the simulation, we observe that awareness, vaccine and therapy are good measure which can be used to effectively control HBV-HDV co-infection in a population. However, awareness and vaccine are better control strategies than therapy. Hence, these simulation results provide the best framework for the control of Hepatitis B virus-Hepatitis D virus (HBV-HDV) co-infection in a population, and effective control of HBV implies effective control of HBV-HDV co-infection in a population.

\section{References}

[1] H. Fejza, S. Telaku, Prevalence of HBV and HCV among blood donors in Kosovo, Virol. J., 13, (2009), 6-21.

[2] S. A. Kafi-abad, H. Rezvan, H. Abolghasemi, Trends in prevalence of hepatitis B virus infection among Iranian blood donors, 1998-2007. Transfus Med., 19(4), (2009), 189-94

[3] M. Rizzetto, G. Verme, S. Recchia, Immunflorescence detection of a new antigen-antibody system (delta-antidelta) associated to hepatitis virus in the liver and serum of HBsAg carriers, Gut., 18, (1977), 996.

[4] A. Smedile, A. Ciancio, M. Rizzetto, Hepatitis D virus. In: Richman, D.D, Whitley, R.J, Hayden, F.G, eds. Clinical virology. Washington, DC: ASM Press, (2002), 1227-1240.

[5] S. I. Friedman, Seminars in medicine of the Beth Israel Hospital, Boston. The cellular basis of hepatic fibrosis. Mechanisms and treatment strategies, England Journal of Medicine; 328, (1993), 1828-1835.

[6] P. Farci, Delta hepatitis: an update, J. Hepatol., 39, Suppl., 1, (2003), 212-9.

[7] S. A. Hughes, H. Wedemeyer, P. M. Harrison, Hepatitis delta virus, Lancet, 378, (2011), $73-85$.

[8] M. Rizzetto, G. Verme, Delta hepatitis-present status, J. Hepatol., 1, (1985), 187-93.

[9] J. M. Taylor, Hepatitis delta virus, Virology, 5; 344(1), (2006), 71-6.

[10] S. M. Alavian, S. H. Alavian, Hepatitis D virus infection; Iran, Middle East and Central Asia, Hepatitis Monthly, 5, (2005), 137-143.

[11] R. Esmaeili, S. M. Alavian, B. Hajibeigi, E. Sabouri, R. Edalat, A. Adeli, Phylogenetic analysis of twenty-six cases of hepatitis delta virus isolates in Tehran, Iran, Hepat Mon, 9(3), (2009), 196-200.

[12] Z. Abbas, W. Jafri, S. Raza, Hepatitis D: Scenario in the Asia-Pacific region, World J. Gastroenterol, 7, 16(5), (2010), 554-62.

[13] S. M. Alavian, Unthought of Problems Regarding Hepatitis D Virus Infection, Hepat Mon., 10(2), (2010), $77-79$.

[14] R. O. Aja, D. Omale, G. C. E. Mbah, Sensitivity Analysis of the Mathematical Model on the Control of HBV-HDV co-infection Transmission Dynamics in a Given Population, Journal of the Nigerian Association of Mathematical Physics, 39, (2017), $457-470$.

[15] R. O. Aja, T. I. Chinebu, E. O. Eze, On the Stability of Hepatitis B Virus-Hepatitis D Virus (HBV-HDV) co-infection with Controls in a Dynamic Population, International Journal of Advances in Mathematics, 2019(2), (2019), 17-30.

[16] H. W. Hethcote, The Mathematics of Infectious Diseases, SIAM REVIEW, 42(4), (2000), 599-653.

[17] S. Abdulrahman, N. I. Akinwande, O. B. Awojoyogbe, U. Y. Abubakar, Sensitivity Analysis of the parameters of a Mathematical Model of Hepatitis B virus transmission, Universal Journal of Applied Mathematics, 1(4), (2013), 230-241.

[18] I. K. Adu, A. Y. Aidoo, I. O. Darko, E. O. Frimpong, Mathematical Model of Hepatitis B in the Bosomtwe District of Ashanti Region, Ghana Applied Mathematical Sciences, 8(64), (2014), 3343 - 3358.

[19] L. Zou, W. Zhang, Modelling the transmission dynamics and control of hepatitis B virus in China, Journal of Theoretical Biology, 10, (2009), 1-9.

[20] A. R. Kimbir, T. Aboiyar, O. Abu, E. S. Onah, Simulation of a Mathematical Model of Hepatitis B virus Transmission Dynamics in the presence of vaccination and treatment, Mathematical Theory and Modelling, 4(12), (2014). 